\title{
A Systematic Review of Hyaluronidase-Assisted Subcutaneous Fluid Administration in Pediatrics and Geriatrics and Its Potential Application in Low Resource Settings.
}

Kelsey Wilhelm MSIV, Dawn Barcellona, MD. University of Arizona College of Medicine-Phoenix

\begin{abstract}
The role of enzyme-assisted subcutaneous fluid administration (EASFA) in treating mild to moderate dehydration has been studied in pediatrics, geriatrics, and palliative care. The objective of this systematic review was to evaluate if EASFA is a successful and effective technique to utilize in the treatment of these populations. Outcomes measured in both the intravenous and subcutaneous groups included rate of fluid infusion and number of attempts to successful catheter placement. The authors found that EASFA is a comparable alternative to intravenous fluids in regards to infusion rates in both pediatric and elderly patients with mild to moderate dehydration. Additionally, the higher rate of successful placement of the subcutaneous catheter may prove useful in select populations and low resource settings.
\end{abstract}

\begin{abstract}
Obtaining IV access in a dehydrated pediatric or geriatric patien can be difficult even for the well trained healthcare provide. Subcutaneous fluid administration does not require venous access and has a low risk of infection, but is relatively underutilized in developing countries. Crystalloid fluids are able to be infused into the subcutaneous tissue after administering the hyaluronidase enzyme. This enzyme hydrolyzes the components of the extracellular matrix, increasing tissue permeability and absorption. Hylenex is a relatively new enzyme recombinant of human hyaluronidase that provides fewer side effects than the previous animal derived enzymes. The process involves injecting the enzyme into the desired site, placement of a catheter into the subcutaneous tissue, and infusing the desired fluids.
\end{abstract}

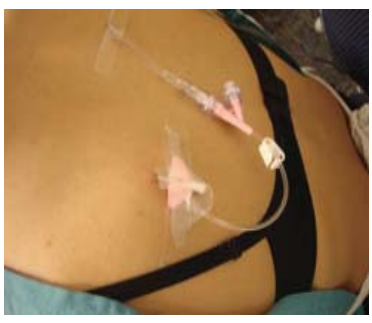

igure 1: An example EASFA using butterfly catheter in inferior shoulder
A review of relevant literature from MEDLINE and Cochrane Library from January 1950 to December 2015 was completed. Authors reviewed studies and selected relevant articles based on inclusion criteria

a. Evaluation of infusion rates and volume infused $O R$

b. Assessment of successful placement of catheter AND

c. Pediatric or elderly population

Statistical analysis was completed using Cohen's D and odds ratios with $95 \%$ confidence intervals.

\section{Included Studies}

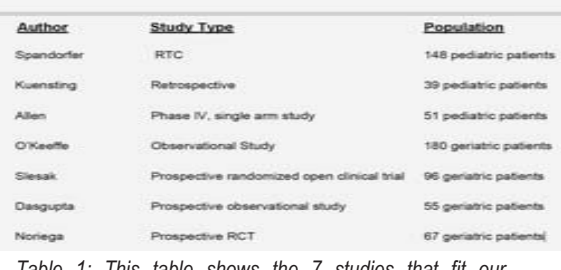

Table 1: This table shows the 7 studies that fit our review.

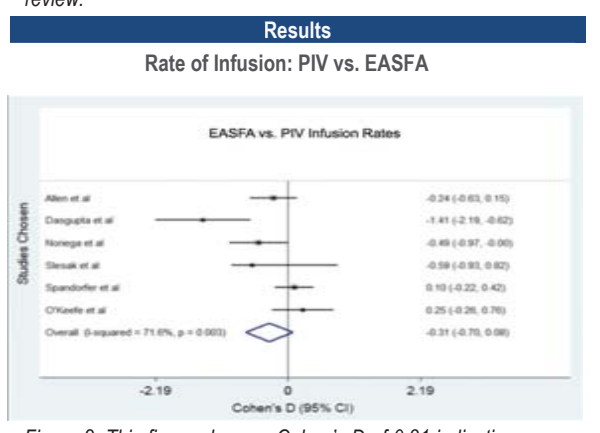

Figure 2: This figure shows a Cohen's D of 0.31 indicating a small effect size of IV over EASFA.
Number of attempts to successful catheter placement: PIV vs. EASFA

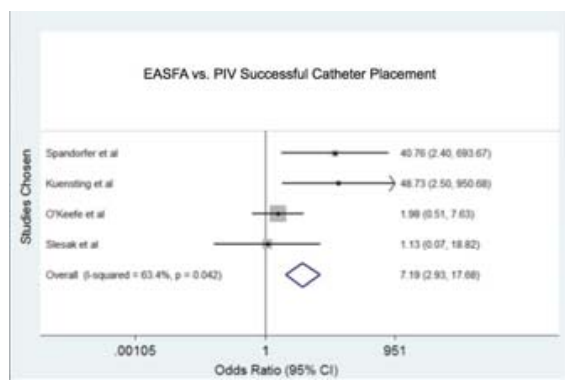

Figure 3: This figure shows a pooled odds ratio of 7.19 Fomering the subcutaneous catheter.

The first outcome measured was the rate of infusion of IV The first oulcome measued wh EASFA. rates of nusion whin the IV group versus 1 . EASFA group.

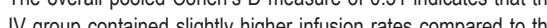

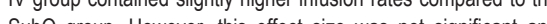
SubQ group. How considered small. In the studies that showed an IV infusion rate

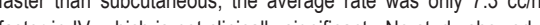
differ in diterence of more than 25 celh faster in the IV group. thestingly, the Spandorfer RTC that showed an infusion rate

Four articles were used to analyze successful catheter placement in the IV and the subcutaneous groups. All of the articles show an increased likelinood of a successful catheter placement using the EASFA method. The overall pooled odds atio shows a 7 times higher likelihood of successful placement within the SubQ group compared to the IV group. This data seems to fit with what we intuitively know, placing subcutaneous catheter is achieved with far more success than catheter placement.

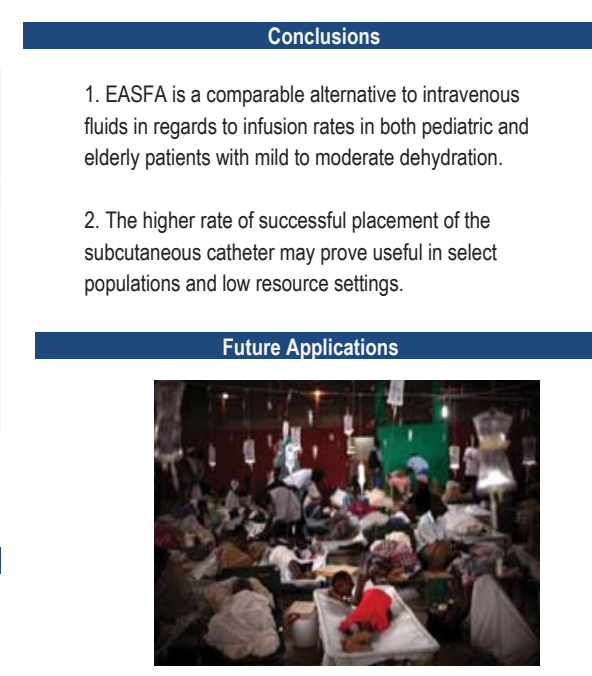

Every year, hundreds of thousands of children and adults die from dehydration secondary to diarrheal illnesses around the world. We know that prompt fluid administration is key to saving these lives. The application of this procedure could be revolutionary especially in low resource settings where there is a lack of trained healthcare personnel to administer intravenous fluids. The ease of catheter placement shows the feasibility to train lay community members to administer fluids when medical care or transport is not readily available. Additionally, this could be considered during a disaster event, cholera epidemic, or anytime patient needs overwhelm the resources of the healthcare system.

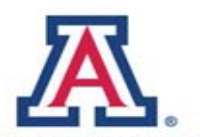

COLLEGE OF MEDICINE

THE UNIVERSITY
PHOENIX 